We appreciate the response to this publication feature and welcome all contributions. Contributions may be sent to José A. Mascorro, our Technical Editor, at his e-mail address: jmascor@tulantedu. José may also. be reached at the Department of Structural and Cellular Biology, Tulane University Health Sciences Center, 1430 Tulane Ave, New Orleans, LA 70112 and Ph: (504) 584-2747 (504) 584-1687

\section{Use of Fishing Weight Putty for Quickly Mounting SEM Specimens}

\author{
Hans Stephenson and Mark Gabel \\ Black Hills State University \\ markgabel@bhsu.edu
}

Projects that require viewing dozens or hundreds of specimens often include countless hours for specimen preparation. Specimens are often affixed to metal or carbon stubs with conductive tape, paint or paste (Rampley, 1976; Witcomb, 1981). The use of conductive paint or paste requires substantial mounting and drying times prior to coating with conductive metal or carbon and observation in a scanning electron microscope (SEM). We here describe a simpler protocol for mounting specimens to expedite specimen preparation.

During a recent study, where cryofractured salmon egg membranes were mounted on edge to view transverse sections, we needed an expedient method of specimen mounting to quickly view hundreds of samples. We experimented with mounting specimens in metal putty, a product used by fishermen for weighting fishing line. The methodology described here solved some of the problems of messy specimen mounting and eliminated the need to wait for curing before coating and observation.

\section{MOUNTING SPECIMENS USING METAL PUTTY}

The procedure for mounting specimens in lead or tungsten putty is extremely simple and time efficient. An adhesive conductive carbon tab was affixed to the top of a clean, conductive aluminum or carbon stub prior to placing the putty on the stub. It is possible to mount specimens in metal putty either vertically (perpendicular) or horizontally (parallel) to the stub surface. To mount specimens vertically, a piece of the putty about $1 / 2$ to $1 \mathrm{~cm}$ in diameter (about the size of a pea) was rolled between gloved thumb and index fingers to form a small "rope" about the length of the diameter of the carbon tab. The putty was pressed firmly onto the carbon tab. A razor blade was used to make several incisions into the putty rope roughly $3 / 4$ the depth of the rope. One specimen was placed into each crevice created by the incisions. After all the specimens were placed in their respective crevices, the putty rope was gently pressed on either side of the specimen with a small pair of forceps. By doing this, the putty firmly contacts the specimen thereby securing it to the stub. To mount specimens horizontally (i.e. parallel to the stub surface) the putty was formed into a small disc approximately equal to the size of the carbon tab. After the putty disc was pressed onto the carbon tab, the specimen can be gently pressed into the putty. The putty should seal around the specimen, thereby securing it to the stub. The samples are immediately ready for sputter coating and observation.

\section{ADVANTAGES TO USING PUTTY AND OTHER CONSIDERATIONS}

Advantages to mounting specimens with putty include: (1) the ease of manipulation or repositioning specimens. (2) The cost of mounting specimens with metal putty is low. A $28 \mathrm{~g} \mathrm{(1oz)} \mathrm{pack-}$ age of metal putty sufficient to mount hundreds of samples costs approximately $\$ 4$ to $\$ 5$. The putty is extremely dense and remains malleable under a wide variety of conditions and is therefore reusable. We have not noticed a loss of malleability after numerous hours of exposure to electrons from a high energy electron beam (accelerating voltage $=20 \mathrm{KeV}$ ) in either high or low vacuum applications. (3) The mounting putty is reusable. The putty can be spread apart and the old specimen easily removed. The incisions will accept new specimens without any additional preparation. The use of putty to mount specimens can be easily adapted to many specimen types and may prove to be a cost- and time-saving replacement for other mounting media. (4) Metal putty does not require volatile solvents and therefore does not "capillary" into porous samples, change the texture, or dissolve the surface layers of specimens. (5) We have had no charging problems using either high vacuum or low vacuum modes in the microscope chamber. Aperture or column contamination from using the dense metal putty has not been observed.

In some states, especially in the northeastern U.S. and in Canadian parks, lead putty is not readily available locally since restrictions have been placed on the use of lead in fishing for fresh water species because of the detrimental effects of lead on waterfowl. The tungsten putty, which is about $30 \%$ more dense than lead putty, is generally available in these areas and both are available through numerous fishing supply houses as well as through internet suppliers.

\section{REFERENCES}

Rampley, D.N. 1976. The effect of the electron beam on various mounting and coating media in the SFM. II. Journal of Microscopy 107: 99-102.

Witcomb, M.J. 1981. The suitability of various adhesives as mounting media for scanning electron microscopy. Journal of Microscopy 121:289-308.

\section{ACKNOWLEDGEMENTS}

The authors appreciate the financial support of the Biomedical Research Infrastructure Network and the Faculty Research Committee of Black Hills State University. We thankfully acknowledge the suggestions by Dr. A. Gabel, Black Hills State University and D. Kessel, University of Iowa.

\section{A Homemade Vacuum Forceps For Mounting Small SEM Samples}

\author{
Sol Sepsenwol \\ University of Wisconsin, Stevens Point \\ ssepsenw@uwsp.edu
}

Very small samples (under $1 \mathrm{~mm}$ ) prepared for SEM viewing can be sprinkled randomly on an adhesive-coated stub, or manipulated into position with various tools (fine forceps, pointed stick, cotton applicator, hair brush) under a magnifier or stereo microscope. None of these methods allows reliable, damage-free control over the orientation of the sample. Here follow plans for a simple, portable "vacuum forceps" to create a mild vacuum through a micro-fine tip to hold and orient SEM samples as they are applied to the adhesive. It is also useful for sorting samples. Commercial vacuum forceps with on-board vacuum range in price from $\$ 500$ to $\$ 900$. This unit was built from new parts for under $\$ 100$.

The vacuum pump is from a portable motorized pipetter, such as the Drummond Scientific Portable Pipet Aid ${ }^{\star}$, or Mano- 